\title{
Volumetric Lagrangian particle tracking measurements of jet impingement on convex cylinder
}

\author{
Mirae Kim ${ }^{1}$, Daniel Schanz ${ }^{2}$, Matteo Novara ${ }^{2}$, Andreas Schröder ${ }^{2}$, Eunseop Yeom ${ }^{1}$, Kyung Chun Kim ${ }^{1}$ \\ ${ }^{1}$ School of Mechanical Engineering, Pusan National University, 46241 Busan, South Korea \\ ${ }^{2}$ Institute of Aerodynamics and Flow Technology, German Aerospace Center (DLR), 37073 Göttingen, Germany
}

Impinging jets are widely used for heat and mass transfer because they are applicable to any type of body and can be easily implemented. They are also used in various industrial fields and design techniques. Previous research investigated e.g. a jet impinging onto a flat surface. However, since most of the mechanical parts have curvature on their surface, it is necessary to study more detailed properties of the jet impinging on a curved surface using advanced measurements. Therefore, in this study, three-dimensional flow structures of a round jet impinging on a convex cylinder surface were measured using volumetric Lagrangian particle tracking (LPT). The experimental setup is shown in Fig 1. The measurement model was a circular cylinder $(\mathrm{D}=52 \mathrm{~mm})$ and a round jet nozzle $(\mathrm{d}=3 \mathrm{~mm})$, which was inserted into a 16-faces glass water tank. Oragasol polyamide particles with a mean diameter of $40 \mu \mathrm{m}$ was used as seeding particles. Four Phantom v2640 high speed cameras were used to capture particle images, operated at $4 \mathrm{kHz}$. For the volume illumination two arrays of high-power white LEDs were used and focused by two large 1,000 mm focal length lenses. Finally, rectangular passe-partouts limited the common illuminated volume to about $80 \times 160 \times 50 \mathrm{~mm}^{3}$. Impingement angles $(\alpha)$ of the jet were 0 and 45 degrees, the distance between the jet and the cylinder surface was fixed at $12 \mathrm{~mm}$, and the Reynolds number based on the jet diameter was 17,875. The measuring area consists of half of the lateral cylinder and the wake area below the cylinder. In order to improve experimental accessibility, the field of view was moved $10 \mathrm{~mm}$ in the $\mathrm{X}$ direction in the case of an impingement angle of $0^{\circ}$ (see Fig. 1). Optical calibration of the imaging system was required to accurately triangulate the position of particles from all $2 \mathrm{D}$ images to $3 \mathrm{D}$ space. A two-plane calibration plate was placed in the cylinder position. Geometrical calibration was refined using volume self-calibration [1] and calibration of the optical transfer function [2]. Lagrangian particle tracking process was performed based on the Variable Time-step Shake-The-Box (VT-STB) algorithm (see [3, 4] or Schanz et al. ISPIV 2021), due to the high dynamic velocity range. The method showed distinct advantages in the reduction of ghost tracks and tracking accuracy over standard processing. Using the FlowFit-algorithm [5], the unstructured particle data containing position, velocity and acceleration is interpolated onto a structured grid of cubic B-splines with $0.7 \mathrm{~mm}$ spacing, while applying physical regularizations. The resulting continuous 3D function is sampled on a 3D Cartesian grid with $0.42 \mathrm{~mm}$ step width. The Lagrangian tracks and assimilated time-resolved 3D3C velocity and pressure fields confirmed that the curved wall jet spreads widely in spanwise direction after impingement, then merges to the jet centerline more downstream (Fig. 2). Three-dimensional entrainment occurs around the curved wall jet. An impinging jet flow with $\alpha=45^{\circ}$ along the cylinder wall shows the Coanda effect with a separation delay at a cylindrical angle of about $180^{\circ}$. However, for an impinging jet flow with $\alpha=0^{\circ}$, flow separation occurs quickly and the flow takes the form of a concentric circle. The ensemble-averaged 3D flow fields near the cylinder wall are shown in Fig. 3. Along the streamwise wall jet flow, the streamwise vorticity was nearly zero because the attached curved jets no longer spread laterally, and were completely randomly generated even in the presence of a streamwise vortex structure in the instantaneous flow field. Since the lateral momentum flux is relatively low compared to the streamwise momentum flux, the gap between the vortex pair structures when $\alpha=45^{\circ}$ is closer than that of $\alpha=0^{\circ}$. The shape of the mean spanwise vorticity structure appeared in a semicircle at $\alpha=0^{\circ}$, and a V-shape at $\alpha=45^{\circ}$. The mean velocity distributions on the cylinder surface can be compared with the cooling area of the surface temperature field results in Kim et al. [6]. 

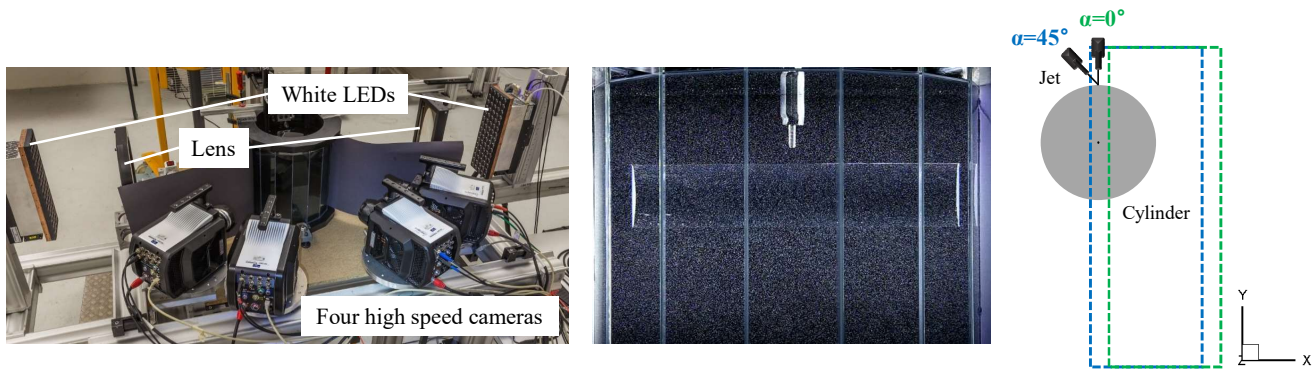

Fig. 1 Left: Experimental setup for volumetric LPT measurements, Middle: Submerged cylinder and jet nozzle, Right: Field of view schematic in side view (XY plane) with blue dashed line at $\alpha=45^{\circ}$ and green dashed line at $\alpha=0^{\circ}$

(a) $\alpha=0^{\circ}$

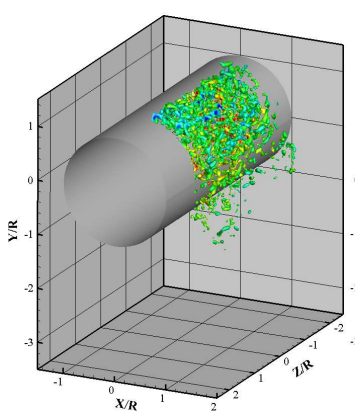

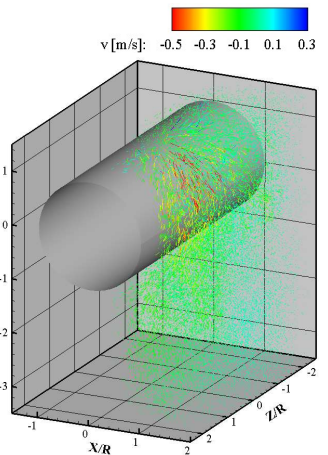

(b) $\alpha=45^{\circ}$

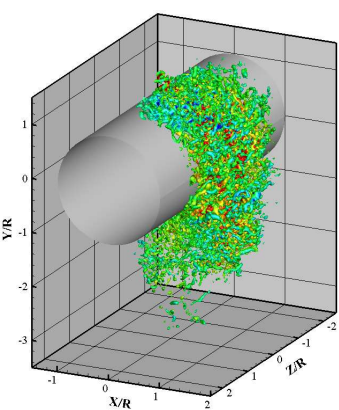

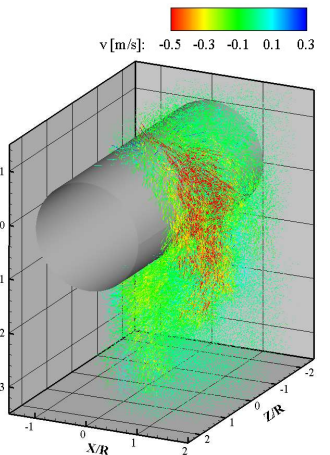

Fig. 2 Instantaneous Lagrangian particle tracks and $Q$ structures $\left(10,000 \mathrm{~s}^{-2}\right)$ colored by v-velocity on convex cylinder

(a) $\alpha=0^{\circ}$
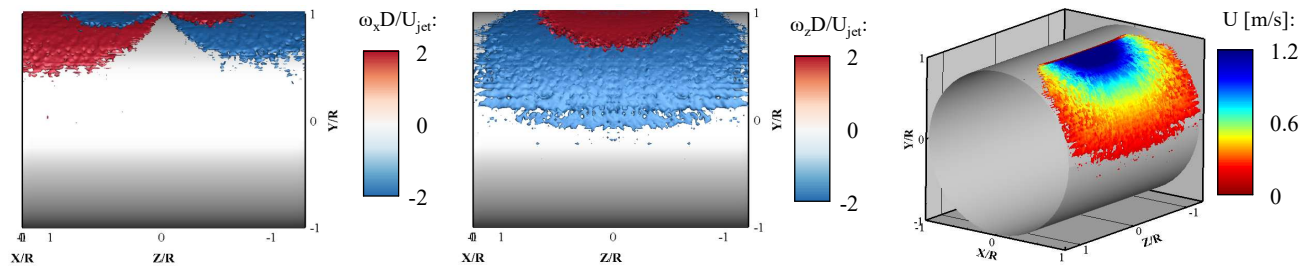

(b) $\alpha=45^{\circ}$
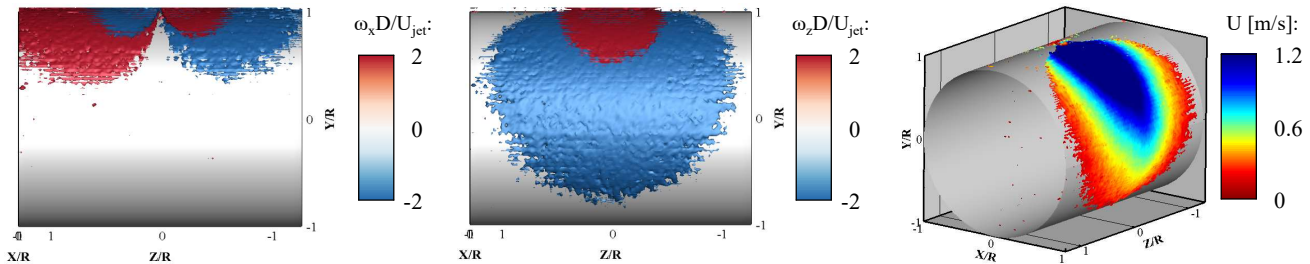

Fig. 3 Ensemble-averaged streamwise and spanwise vortex structures and mean velocity distributions on cylinder surface

\section{References}

[1] Wieneke, B. (2008) Volume self-calibration for 3D particle image velocimetry. Exp. Fluids, 45(4), 549-556.

[2] Schanz, D., Gesemann, S., Schröder, A., Wieneke, B., \& Novara, M. (2012) Non-uniform optical transfer functions in particle imaging: calibration and application to tomographic reconstruction. Meas. Sci. Technol., 24(2), 024009.

[3] Schanz, D., Gesemann, S., \& Schröder, A. (2016) Shake-The-Box: Lagrangian particle tracking at high particle image densities. Exp. Fluids, 57(5), 70.

[4] Schanz D., Novara M. \& Schröder A. (2020) Shake-The-Box particle tracking with variable time-steps in flows with high velocity range (VT-STB). 3rd Workshop on DA \& CFD Processing for PIV and LPT.

[5] Gesemann, S., Huhn, F., Schanz, D., \& Schröder, A. (2016, July) From noisy particle tracks to velocity, acceleration and pressure fields using B-splines and penalties. In $18^{\text {th }}$ LXLASER, Lisbon, Portugal (pp. 4-7).

[6] Kim, M., Kim, D., Yeom, E. (2020) Measurement of three-dimensional flow structure and transient heat transfer on curved surface impinged by round jet. Int. J. Heat Mass Transfer 161, 120279.

\section{Acknowledgements}

This work was supported by the National Research Foundation of Korea(NRF) grant funded by the Korea government(MSIT) (No. 2021R1C1C2009287). This work was partly supported by the Deutsche Forschungsgemeinschaft (DFG) through Grant No. SCHR 1165/5-1 as part of the Priority Programme on Turbulent Superstructures (DFG SPP 1881, 2nd period). 